\title{
Cognitive function of patients with adult moyamoya disease.
}

\section{AUTHOR(S):}

Araki, Yoshio; Takagi, Yasushi; Ueda, Keita; Ubukata, Shiho; Ishida, Junko; Funaki, Takeshi; Kikuchi, Takayuki; Takahashi, Jun C; Murai, Toshiya; Miyamoto, Susumu

\section{CITATION:}

Araki, Yoshio ... [et al]. Cognitive function of patients with adult moyamoya disease.. Journal of stroke and cerebrovascular diseases 2014, 23(7): 1789-1794

\section{ISSUE DATE:}

2014-08

URL:

http://hdl.handle.net/2433/189552

\section{RIGHT:}

(C) 2014 National Stroke Association. Published by Elsevier Inc.; この論 文は出版社版でありません。引用の際には出版社版をご確認ご利用く ださい。; This is not the published version. Please cite only the published version. 


\section{Cognitive Function of Patients with Adult Moyamoya Disease}

Yoshio Araki, $\mathrm{MD}, \mathrm{PhD}^{1}$, Yasushi Takagi, $\mathrm{MD}, \mathrm{PhD}^{1}$, Keita Ueda, $\mathrm{MD}, \mathrm{PhD}^{2}$, Shiho

Ubukata, MHSc ${ }^{2}$, Junko Ishida, OTR ${ }^{3}$, Takeshi Funaki, MD ${ }^{1}$, Takayuki Kikuchi, MD,

$\mathrm{PhD}^{1}$, Jun C Takahashi, MD, $\mathrm{PhD}^{1}$, Toshiya Murai, $\mathrm{MD}, \mathrm{PhD}^{2}$, Susumu Miyamoto, MD, $\mathrm{PhD}^{1}$

1. Department of Neurosurgery, Kyoto University Graduate School of Medicine

2. Department of Psychiatry, Kyoto University Graduate School of Medicine

3. Department of Rehabilitation, Kyoto University Hospital

Corresponding Author:

Yoshio Araki, MD, PhD

Department of Neurosurgery, Kyoto University Graduate School of Medicine, 54

Kawahara-cho, Shogoin, Sakyo-ku, Kyoto, Japan 606-8507

E-mail: yaraki@kuhp.kyoto-u.ac.jp 
Phone: $+81-75-751-3459$

Fax: $+81-75-752-9501$

Running title: Cognitive Function in Adult Moyamoya Disease

Key words: Moyamoya disease; cognitive impairment; neuropsychological tests; adult 


\section{Introduction}

Moyamoya disease is an uncommon cerebrovascular condition characterized by progressive occlusion of bilateral internal carotid arteries, and is known to cause strokes in relatively younger people.[1,2] Several efforts to identify its pathogenesis have recently detected gene mutations and deletions that make people susceptible to the familial form of the disease, and further investigation might clarify the direct mechanisms underlying the disease.[3-6] Extracranial-intracranial bypass surgery has been established as an effective neurosurgical intervention that increases cerebral blood flow (CBF) and guards against ischemic attacks. However, difficulty with social independence accompanied by cognitive impairment has recently been recognized as an important unsolved social issue faced by patients with adult moyamoya disease.[7] These patients are physically independent in daily life, but economically dependent because cognitive impairment leads to difficulty obtaining vocational skills. Here, we define the status of these patients as "difficulty with social independence." Generally, cognitive impairment has been described as a neuropsychological sequela occurring after strokes that manifests as disturbances in memory, attention, performance, and 
social behavioral in pediatric cases.[8, 9] However, recent reports have focused on adult cases with neurocognitive impairment even without radiological evidence of major stroke. $[7,10]$ Nakagawara et al. indicated that even if infarction has not yet occurred, brain dysfunction was associated with persistent hemodynamic compromise in the medial frontal lobes that can be visualized using $\left[{ }^{123}\right.$ I]iomazenil (IMZ)-single photon emission CT (SPECT). This technique has the potential to become a tool for diagnosing cognitive impairment in adult moyamoya patients who do not show major abnormalities on CT scans or magnetic resonance imaging (MRI). In contrast, a common methodology for neuropsychological evaluation of these patients is yet to be determined, even to the extent that which questions to include remains undecided. Because previous studies have selected considerably different tasks for this evaluation, results have been unsurprisingly in consistent.[10-12] Therefore, we address this concern by administering structured tests to two groups of adult moyamoya patients, one with difficulty in social independence, and the other without.

\section{Materials and Methods}




\section{Participants}

Ten patients with neuroradiologically confirmed adult moyamoya disease ( 3 men and 7

women; mean age: 34.2 years; range, 19-51 years) participated in this study. Since this

survey was formed by completely anonymous retrospective information, this study did

not have the ethics committee approval. All subjects were proficient in Japanese. To

identify specifics regarding neuropsychological assessment in moyamoya patients who

have difficulty with social independence, the 10 patients were divided into two groups.

Group 1 comprised five patients without difficulty in social independence. The subjects

in this group had a higher educational background without need for special education

programs, better socioeconomic status, and did not need public support. Group 2

comprised five patients who had difficulty with social independence. Two of the five

patients required a special education program, and all were socioeconomically

disadvantaged and needed public support. The mean duration of the disease was 9.1

years. Only one patient had a history of small intracerebral hemorrhage (periventricular

region) at onset. Other patients had histories of transient ischemic attacks or minor

ischemic strokes. MRI revealed these minor strokes in four patients, while the 
remaining subjects showed no abnormalities in the radiological assessment. No subjects showed radiological abnormality evidenced by an ischemic lesion affected by more than two cortical arteries. $\left[{ }^{123}\right.$ I]iodoamphetamine (IMP)-SPECT showed one case of resting-state CBF impairment in Group 1 and three cases in Group 2. Cerebrovascular reserve (CVR) impairment was found in nine of the ten cases. Revascularization surgery comprising superficial temporal artery-middle cerebral artery bypass was performed in nine of the ten patients and their preoperative symptoms were relieved. All patients were physically independent, with modified Rankin Scale scores no greater than 2 at the time of study inclusion. Table 1 and Table 2 summarize the clinical characteristics and radiological features of each patient group.

\section{Neuropsychological Assessment}

Basic cognitive ability was evaluated using the Wechsler Adult Intelligence Scale-Third

Edition (WAIS-III) to assess intelligence, the Wechsler Memory Scale-Revised

(WMS-R) to assess memory[13, 14], and supplemental subtests for each task. Several frontal-functioning tests were also administered to detect specific neuropsychological 
deficits associated with adult moyamoya disease that co-occurs with difficulty in social independence. The Frontal Assessment Battery (FAB) tested general frontal cognitive ability. The Trail Making Test Part A (TMT-A) assessed speed of information processing $[15,16]$ and the Trail Making Test Part B (TMT-B) and the Wisconsin Card Sorting Test assessed executive ability.[16, 17] The Go/No-Go and No-Go/Go tasks were used to measure response inhibition[18] and the Apathy Scale measured the extent of apathy. The Reading the Mind in the Eyes (Eyes) task is a theory-of-mind task that was given to examine the ability to infer the mental status of others.[19]

\section{Data Analysis}

To identify group differences regarding clinical profiles and neuropsychological tasks, a univariate analysis was performed. P-values were calculated based on the two-tailed $t$-test for parametric data and the Mann-Whitney $U$ test for non-parametric data. Next, to determine which factors contributed to the differentiation between groups, a discriminate analysis was applied to the data set. A predictive model was then constructed following a stepwise variable selection procedure. Finally, the contribution 
rate that discriminated between the groups and the expected classification rate were calculated along with their F- and P-values. These statistical data were generated using JMP software, Version 10.0.2 (SAS Institute Inc., Cary, NC, USA). A p-value less than 0.05 was considered statistically significant.

\section{Results}

The mean scores for clinical variables and neuropsychological assessments of each patient group are given in Table 3. The mean age of Group 1 was substantially higher than that of Group 2, but the disease duration for each group was not significantly different. Group 1 also had significantly higher mean scores than Group 2 for intelligence functions including subtests for basic cognitive abilities. In contrast, scores for memory functions showed significant differences in three subtests (General Index, Attention/Concentration Index, and Delayed Index) between groups. Although scores were equivalent between groups on a number of tasks that assessed frontal lobe functions, those obtained from the TMT-B test were significantly higher in Group 2, while those from the Eyes test were significantly higher in Group 1. After loading all 
data from neuropsychological tasks into statistical software, a discriminate analysis was performed. The result indicated that the Working Memory (a WAIS-III subtest) and Eyes tasks contributed significantly to the discrimination of the groups (Table 4).

\section{Discussion}

The present study demonstrated that impairments were mainly in intelligence and memory function. Additionally, some frontal lobe function was particularly affected in adult moyamoya patients with difficulty in social independence.

\section{Evaluation of the results in this study}

\section{Neuropsychological examination}

Recent work using IMZ-SPECT has demonstrated the association between cortical neuron loss in bilateral frontal medial cortices and cognitive dysfunction. Considering that evidence, we have adopted several tasks to examine frontal lobe functions. To date, this is the first adoption of this comparative method regarding cognitive function of moyamoya disease.[7, 9-12, 20-22] Therefore, the data presented here are novel and not 
comparable with prior studies. A definition of "neurocognitive dysfunction" using the evaluations from all the proposed tasks was not presumed in considering the objective of this study.

\section{Intelligence and Memory function}

On measures of intelligence abilities using WAIS-III and its subtests, mean scores from all patients in Group 2 were found to be lower than that of Group 1. Previous research has demonstrated loss of intellectual functions in pediatric-onset cases.[8, 9, 20, 23, 24] Our results from Group 2 were consistent with those based on the age of onset. In contrast, the mean level of intelligence ability in Group 1 was preserved. These data are consistent with a report suggesting cognition in adult moyamoya cases is relatively spared.[22] The proportion of gainfully employed subjects in that report (84\%) is comparable that in this one $(80 \%)$. Interestingly, within Group 1, Working Memory scores were lower compared to scores from other WAIS-III subtests. This may be specifically related to adult moyamoya cases that do not include difficulty with social independence. The underlying CBF impairment or neuronal loss induced by prolonged 
hemodynamic compromise could lead to a mild disorder in intellectual functioning that manifests in working-memory deficits. However, memory ability assessed by the WMS-R was not different from other abilities, including those assessed by all WMS-R subtests. This highlights the difficulty assessing memory status in the adult moyamoya population, which is still controversial. While Festa et al. have reported an overall memory score 1.1 SDs below the mean of healthy individuals,[12] other reports have shown memory to be unaffected in adult moyamoya subjects.[11, 22] Usually, a lack of memory impairment associated with spared hypoperfusion in the medial temporal lobe is characteristic of moyamoya disease. However, our results showing impairment within Group 2 on three subtests of the WMS-R could not be explained from the SPECT data, indicating specific hypoperfusion in the rest state and impaired CVR in the medial temporal lobe. This point remains unresolved, whereas memory function may be associated not only with the medial temporal lobe but also with wide-spread subcortical neuronal connections.

\section{Frontal lobe functions}


An extensive focus on frontal lobe function has not yet been taken by previous research regarding moyamoya disease. CBF and IMZ studies have shown that antero-medial frontal cortices fed by anterior circulation develop blood insufficiencies.[7, 25] For this reason, several neuropsychological test batteries to evaluate frontal lobe functioning in relation with hemodynamic compromise were employed for this preliminary study. Among these batteries, only scores from the TMT-B and Eyes tasks were shown to be statistically lower in Group 1 compared with Group 2. The TMT-B can estimate frontal lobe function in terms of problem solving and motor planning.[26] Performance on this test is known to be poor in adult patients with moyamoya disease, $[12,22]$ and results from Group 2, required time to complete the task was longer than Group 1, were compatible with these other studies. Theory-of-mind tasks examine one's ability to infer the mental status of others. Here we employed the revised version of the Reading the Mind in the Eyes test.[19, 27] This test had been given to patients with other kinds of psychiatric disorders, and recent neuroimaging studies of normal subjects indicate that performing the Eyes task activates linked brain regions including the medial prefrontal cortex, the orbitofrontal cortex, the amygdala, the temporal poles and the superior 
temporal sulcus.[28] Hirao et al. has demonstrated that the mean accuracy in the Eyes task generated by schizophrenic patients was significantly lower than that of normal subjects.[27] Furthermore, they provided a correlation analysis between Eyes-task impairment and structural alterations using Voxel-based morphometry, which indicated specific regional abnormalities in the left ventrolateral prefrontal cortex of schizophrenia patients. To our knowledge, this is the first report to show deficiency in theory-of-mind ability in patients with moyamoya disease. Though we did not include a structural study, long-term chronic hypoperfusion in the anterior circulation could produce a dysfunction in medial and lateral regions of the anterior frontal lobe, which might induce the theory-of-mind impairment observed in Group 2.

\section{Discriminate analysis}

To determine which neuropsychological tasks can best detect neurocognitive dysfunction in adult patients with moyamoya disease in a clinical setting, we conducted a discriminate analysis using crude data from all neuropsychological tasks. Results showed that the Working Memory and Eyes tasks were the best predictors, and a model 
limited to those tasks successfully classified the patients into two groups. This indicates that these two tasks have the statistical power to diagnose neurocognitive dysfunction in adult patients with moyamoya disease. Impairment of these tasks could be the specific neurocognitive deficits that inflict adult moyamoya patients.

\section{Limitations}

There are several limitations to this preliminary study. First, the definition of "difficulty with social independence" is still unclear, and selecting these patients was not objective, so it may be biased. A structured evaluation system determined through a multi-center study is required. Second, in considering the effect of CBF on neurocognitive function, a history of revascularization surgery should be matched. However, the aim of this study is not to compare neurocognitive function before and after the revascularization surgery, but to collect long-term consequence of neurocognitive function in adult patients with moyamoya disease. Third, the number of patients enrolled in this study was too small. Characteristics such as age, type of onset, radiological abnormality were not matched between groups. However, it is particularly worth nothing that the several group 
differences were revealed even in small set of patients and detailed neuropsychological tasks. This preliminary study would be fundamental data for a large-scale research and contribute to understand the characteristics of cognitive dysfunction in adult patients with moyamoya disease

\section{Conclusions}

This study profiled neurocognitive function in adult patients with moyamoya disease using structured neuropsychological tasks. We showed that a broad range of cognitive functions is disrupted particularly in the patients with difficulty in social independence.

We found that scores from the Working Memory (WAIS-III) and Eyes tasks are a novel clinical approach to detect such disadvantaged subjects even if they lack obvious abnormalities in brain images. Our findings also reveal subtle impairments in intelligence function (Working Memory, WAIS-III) in the socially independent patient population. To obtain sufficiently powered evidence regarding the cognitive deficits reported here, a multi-center prospective study is needed in patients with moyamoya disease. 


\section{References}

[1] Suzuki J, Takaku A. Cerebrovascular "moyamoya" disease. Disease showing abnormal net-like vessels in base of brain. Arch Neurol 1969;20:288-299.

[2] Hoshino H, Izawa Y, Suzuki N. Epidemiological features of moyamoya disease in Japan. Neurol Med Chir (Tokyo) 2012;52:295-298.

[3] Pandey P, Steinberg GK. Neurosurgical advances in the treatment of moyamoya disease. Stroke 2011;42:3304-3310.

[4] Liu W, Morito D, Takashima S, Mineharu Y, et al. Identification of RNF213 as a susceptibility gene for moyamoya disease and its possible role in vascular development.

PLoS One 2011;6:e22542.

[5] Kamada F, Aoki Y, Narisawa A, et al. A genome-wide association study identifies RNF213 as the first Moyamoya disease gene. J Hum Genet 2011;56:34-40.

[6] Araki Y, Yoshikawa K, Okamoto S, et al. Identification of novel biomarker candidates by proteomic analysis of cerebrospinal fluid from patients with moyamoya disease using SELDI-TOF-MS. BMC neurol 2010;10:112. 
[7] Nakagawara J, Osato T, Kamiyama K, et al. Diagnostic imaging of higher brain dysfunction in patients with adult moyamoya disease using statistical imaging analysis for [123I]iomazenil single photon emission computed tomography. Neurol Med Chir (Tokyo) 2012;52:318-326.

[8] Sato H, Sato N, Tamaki N, et al. Chronic low-perfusion state in children with moyamoya disease following revascularization. Childs Nerv Syst 1990;6:166-171.

[9] Matsushima $\mathrm{Y}$, Aoyagi $\mathrm{M}$, Masaoka $\mathrm{H}$, et al. Mental outcome following encephaloduroarteriosynangiosis in children with moyamoya disease with the onset earlier than 5 years of age. Childs Nerv Syst 1990;6:440-443.

[10] Karzmark P, Zeifert PD, Bell-Stephens TE, et al. Neurocognitive impairment in adults with moyamoya disease without stroke. Neurosurgery 2012;70:634-638.

[11] Weinberg DG, Rahme RJ, Aoun SG, et al. Moyamoya disease: functional and neurocognitive outcomes in the pediatric and adult populations. Neurosurg Focus 2011;30:E21.

[12] Festa JR, Schwarz LR, Pliskin N, et al. Neurocognitive dysfunction in adult moyamoya disease. J Neurol 2010;257:806-815. 
[13] Wechsler D. Wechsler Adult Intelligence Scale-Third Edition. San Antonio: The Psychological Corporation, 1997.

[14] Wechsler D. Wechsler Memory Scale-Revised. San Antonio: The Psychological Corporation, 1987.

[15] Dubois B, Slachevsky A, Litvan I, et al. The FAB: a Frontal Assessment Battery at bedside. Neurology 2000;55:1621-1626.

[16] Reitan RM. The Halstead-Reitan Neuropsychological Test Battery. Tucson: Neuropsychology Press, 1985.

[17] Heaton R CG, Talley J, Kay G, et al. Wisconsin card sorting test manual revised and expanded. Odessa: Psychological Assessment Resources, 1993.

[18] Chikazoe J, Jimura K, Hirose S, et al. Preparation to inhibit a response complements response inhibition during performance of a stop-signal task. J Neuroscience 2009;29:15870-15877.

[19] Baron-Cohen S, Wheelwright S, Hill J, et al. The "Reading the Mind in the Eyes" Test revised version: a study with normal adults, and adults with Asperger syndrome or high-functioning autism. J Child Psychol Psychiatry 2001;42:241-251. 
[20] Ohtaki M, Uede T, Morimoto S, et al. Intellectual functions and regional cerebral haemodynamics after extensive omental transplantation spread over both frontal lobes in childhood moyamoya disease. Acta Neurochir (Wien) 1998;140:1043-1053.

[21] Mogensen MA, Karzmark P, Zeifert PD, et al. Neuroradiologic correlates of cognitive impairment in adult Moyamoya disease. AJNR Am J Neuroradiol 2012;33:721-725.

[22] Karzmark P, Zeifert PD, Tan S, et al. Effect of moyamoya disease on neuropsychological functioning in adults. Neurosurgery 2008;62:1048-51.

[23] Ishii R, Takeuchi S, Ibayashi $\mathrm{K}$, et al. Intelligence in children with moyamoya disease: evaluation after surgical treatments with special reference to changes in cerebral blood flow. Stroke 1984;15:873-877.

[24] Imaizumi C, Imaizumi T, Osawa M, et al. Serial intelligence test scores in pediatric moyamoya disease. Neuropediatrics 1999;30:294-299.

[25] Kaku Y, Iihara K, Nakajima N, et al. Cerebral blood flow and metabolism of hyperperfusion after cerebral revascularization in patients with moyamoya disease. $\mathrm{J}$ Cereb Blood Flow Metab 2012;32:2066-2075. 
[26] Zakzanis KK, Mraz R, Graham SJ. An fMRI study of the Trail Making Test. Neuropsychologia 2005;43:1878-1886.

[27] Hirao K, Miyata J, Fujiwara H, et al. Theory of mind and frontal lobe pathology in schizophrenia: a voxel-based morphometry study. Schizophr Res 2008;105:165-174.

[28] Brunet-Gouet E, Decety J. Social brain dysfunctions in schizophrenia: a review of neuroimaging studies. Psychiatry Res 2006;148:75-92. 
Table 1. Summary of clinical characteristics of each patient group

\begin{tabular}{|c|c|c|c|c|c|c|c|}
\hline Case No. & Group & Age, y.o. & Sex & $\begin{array}{c}\text { disease } \\
\text { duration, y }\end{array}$ & $\begin{array}{c}\text { Revascularization } \\
\text { surgery }\end{array}$ & $\begin{array}{c}\text { Special } \\
\text { education }\end{array}$ & $\begin{array}{c}\text { Employment and } \\
\text { economic } \\
\text { independence }\end{array}$ \\
\hline 1 & 1 & 51 & $\mathrm{~F}$ & 1 & bil. STA-MCA+EMS & no & yes \\
\hline 2 & 1 & 42 & $\mathrm{~F}$ & 1 & 1t STA-MCA+EMS & no & yes \\
\hline 3 & 1 & 51 & M & 0 & 1t STA-MCA+EMS & no & yes \\
\hline 4 & 1 & 44 & $\mathrm{~F}$ & 9 & bil. STA-MCA+EMS & no & no \\
\hline 5 & 1 & 28 & $\mathrm{~F}$ & 3 & 1t STA-MCA+EMS & no & yes \\
\hline 6 & 2 & 20 & $\mathrm{~F}$ & 19 & bil. STA-MCA+EMS & yes & no \\
\hline 7 & 2 & 19 & M & 12 & bil. STA-MCA+EMS & yes & no \\
\hline 8 & 2 & 19 & $\mathrm{~F}$ & 11 & bil. STA-MCA+EMS & yes & no \\
\hline 9 & 2 & 25 & M & 0 & no & no & no \\
\hline 10 & 2 & 43 & $\mathrm{~F}$ & 35 & bil. STA-MCA+EMS & no & no \\
\hline
\end{tabular}

Group 1: patients without difficulty in social independence; Group 2: patients with difficulty in social independence; y.o.; years old; y, years; bil., bilateral; STA-MCA, superficial temporal artery-middle cerebral artery bypass; EMS, encephalo-myo-synangiosis 
Table 2. Summary of radiological features of each patient group

\begin{tabular}{|c|c|c|c|c|c|}
\hline \multirow[t]{2}{*}{ Case No. } & \multirow[t]{2}{*}{ Group } & \multicolumn{2}{|l|}{ Lesions on MR imaging } & \multicolumn{2}{|l|}{ SPECT findings } \\
\hline & & minor stroke & bleeding & rest & CVR \\
\hline 1 & 1 & - & - & preserved & impaired in bil. ACA and MCA territory \\
\hline 3 & 1 & 1t. basal ganglia & - & impaired in lt. MCA territory & impaired in lt. MCA territory \\
\hline 4 & 1 & - & - & preserved & impaired in bil. ACA and MCA territory \\
\hline 5 & 1 & - & - & preserved & impaired in bil. ACA territory \\
\hline 7 & 2 & - & - & preserved & impaired in bil. ACA and MCA territory \\
\hline 8 & 2 & bil. occipital and temporal lobe CoI. & - & impaired in bil. PCA territory & impaired in bil. ACA, MCA and PCA territory \\
\hline 9 & 2 & - & - & impaired in rt. ACA and MCA territory & impaired in rt. ACA and MCA territory \\
\hline 10 & 2 & - & - & preserved & impaired in bil. ACA and MCA territory \\
\hline
\end{tabular}


Table 3. Summary of clinical variables and neuropsychological assessments in each group

\begin{tabular}{|c|c|c|c|}
\hline & Group1 (n=5) & Group2 (n=5) & Statistics \\
\hline & Mean (S.D.) & Mean (S.D.) & $p$ \\
\hline \multicolumn{4}{|l|}{ Clinical variables } \\
\hline Age (y.o.) & $43.2(9.4)$ & $25.2(10.3)$ & $0.0273 *$ \\
\hline disease duration (y.) & $2.8(3.6)$ & $15.4(12.9)$ & 0.09 \\
\hline \multicolumn{4}{|l|}{ Intelligence (WAIS-III) } \\
\hline Verbal IQ & $103.6(17.5)$ & $67.2(6)$ & $0.009 * *$ \\
\hline Performance IQ & $95.6(10.7)$ & $60.6(10.5)$ & $0.0086^{* *}$ \\
\hline Full Scale IQ & $100(15.2)$ & $61.4(6.9)$ & $0.009 * *$ \\
\hline Verbal Comprehension & $108.2(19)$ & $72.4(15.4)$ & $0.0088 * *$ \\
\hline Perceptual Organization & $98.6(12.5)$ & $63.6(7.6)$ & $0.009 * *$ \\
\hline Working memory & $91.8(11.2)$ & $61.4(3.3)$ & $0.008 * *$ \\
\hline Processing Speed & $98(8.6)$ & $62.4(12.8)$ & $0.009 * *$ \\
\hline \multicolumn{4}{|l|}{ Memory (WMS-R) } \\
\hline Verbal Index & $99.2(26.9)$ & $69.6(7.2)$ & 0.1161 \\
\hline Visual Index & $111.6(12)$ & 76.8 (28.9) & 0.0749 \\
\hline General Index & $103.2(25.4)$ & $65(14.3)$ & $0.0283^{*}$ \\
\hline Attn./Conc.Index & $100.6(8.9)$ & $68.4(14)$ & $0.009 * *$ \\
\hline Delayed Index & $106(24.3)$ & $64.4(16)$ & $0.0283^{*}$ \\
\hline \multicolumn{4}{|l|}{ Frontal lobe functions } \\
\hline Frontal assessment battery & $16.8(0.8)$ & $16.6(1.1)$ & 0.8266 \\
\hline Trail Making Test A & $35(17.4)$ & $72.8(30.4)$ & 0.0593 \\
\hline Trail Making Test B & $71.8(24.4)$ & $120.4(37.4)$ & $0.0465^{*}$ \\
\hline Wisconsin Card Sorting Test & $3.8(2.3)$ & $3(2)$ & 0.5232 \\
\hline Go/NoGO task & $276.2(39)$ & $330.8(122.5)$ & 0.6015 \\
\hline NoGo/Go task & $99.2(9.2)$ & $105.2(27)$ & 0.754 \\
\hline Apathy Scale & $13.8(2.5)$ & $16.6(3.6)$ & 0.2418 \\
\hline Theory of Mind (Eyes) & $24(3.2)$ & $16.8(1.6)$ & $0.0086 * *$ \\
\hline
\end{tabular}

Group 1, patients without difficulty in social independence; Group 2, patients with difficulty with difficulty in social independence; S.D., standard deviation; y.o., years old; WAIS-III, Wechsler Adult Intelligence Scale-Third Edition; WMS-R, Wechsler Memory Scale-Revised; Attn, Attention; Conc, Concentration; ${ }^{*} \mathrm{P}<0.05$, **P $<0.01$ 
Table 4. Discriminate analysis for prediction model

\begin{tabular}{|c|c|c|}
\hline Neuropsychological test & F value & $P$ value $($ Prob $>\mathrm{F})$ \\
\hline \multicolumn{3}{|l|}{ Intelligence (WAIS-III) } \\
\hline Verbal IQ & 0.807 & 0.404 \\
\hline Performance IQ & 0.282 & 0.614 \\
\hline Full Scale IQ & 0.69 & 0.438 \\
\hline Verbal Comprehension & 0.087 & 0.778 \\
\hline Perceptual Organization & 0.118 & 0.743 \\
\hline Working memory & 16.75 & $0.005 * *$ \\
\hline Processing Speed & 0.033 & 0.861 \\
\hline \multicolumn{3}{|l|}{ Memory (WMS-R) } \\
\hline Verbal Index & 0.014 & 0.911 \\
\hline Visual Index & 0.017 & 0.901 \\
\hline General Index & 0.002 & 0.964 \\
\hline Attn./Conc.Index & 0.139 & 0.723 \\
\hline Delayed Index & 0.143 & 0.719 \\
\hline \multicolumn{3}{|l|}{ Frontal lobe function } \\
\hline Frontal assessment battery & 0.063 & 0.811 \\
\hline Trail Making Test A & 0.792 & 0.408 \\
\hline Trail Making Test B & 1.662 & 0.245 \\
\hline Wisconsin Card Sorting Test & 0.19 & 0.678 \\
\hline Go/No GO task & 0.059 & 0.817 \\
\hline NoGo/Go task & 0.231 & 0.648 \\
\hline Apathy Scale & 1.073 & 0.340 \\
\hline Theory of Mind (Eyes) & 8.636 & $0.022 *$ \\
\hline
\end{tabular}

WAIS-III, Wechsler Adult Intelligence Scale-Third Edition; WMS-R,

Wechsler Memory Scale-Revised; $* \mathrm{P}<0.05, * * \mathrm{P}<0.01$ 